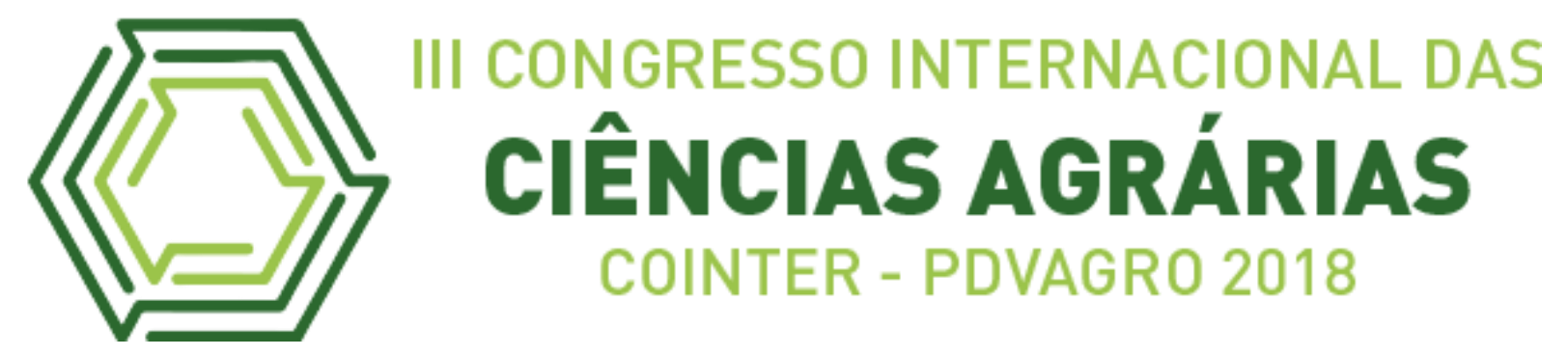

\title{
MEDIÇÃO DE PERDA DE CARGA PRINCIPAL EM UMA MANGUEIRA DE POLIETILENO
}

\section{MEASUREMENT OF MAIN LOAD LOSS IN A POLYETHYLENE HOSE}

\author{
Apresentação: Relato de Experiência \\ Thayane Leonel Alves ${ }^{1}$; Gabriela de Almeida Mourão ${ }^{2}$; José de Arruda Barbosa ${ }^{3}$; \\ Antônio Michael Pereira Bertino ${ }^{4}$; José Renato Zanini ${ }^{5}$ \\ DOI: https://doi.org/10.31692/2526-7701.IIICOINTERPDVAGRO.2018.00746
}

\section{Introdução}

A crescente demanda por alimentos no mundo implícita o aumento da produtividade, uma opção para que isso ocorra é a utilização da irrigação na agricultura. O planejamento de um sistema de irrigação, exige, além dos conhecimentos da cultura, conhecimentos básicos de hidráulica e a perda de carga que ocorre nas tubulações é um dado muito importante para o dimensionamento desse sistema.

A perda de carga, em sistemas de bombeamento de líquidos, é denominada quando ocorre um deslocamento de um líquido de dentro de um tubo e esse deslocamento causa atrito entre o fluido e a parede interna do tubo, ocorrendo-se perda de energia (ANDRADE, [20--?]).

A perda de carga pode ser classificada em perda de carga principal ou distribuída, sendo a que ocorre ao longo da tubulação e perda de carga secundária ou localizada, ocorrendo em pontos distintos da tubulação, sendo eles: tês, joelho, válvulas, curvas, ampliadores, etc. O somatório dessas perdas é muito importante para se projetar um sistema de irrigação, a fim de obter uma melhor escolha técnica e econômica do material a ser utilizado (ECO EDUCACIONAL ,2014).

A perda de carga é determinada pelas seguintes fórmulas (ZANINI, 2017):

\footnotetext{
${ }^{1}$ Mestranda em Agronomia (Ciência do Solo), UNESP - Campus de Jaboticabal - Jaboticabal / São Paulo; E-mail: thayaneleonel@hotmail.com

${ }^{2}$ Mestranda em Agronomia (Ciência do Solo), UNESP - Campus de Jaboticabal - Jaboticabal / São Paulo; E-mail: gabrielamouraodealmeida@gmail.com

${ }_{3}^{3}$ Mestrando em Agronomia (Ciência do Solo), UNESP - Campus de Jaboticabal - Jaboticabal / São Paulo, E-mail: josearruda777@gmail.com

${ }^{4}$ Doutorando em Agronomia (Produção Vegetal), UNESP - Campus de Jaboticabal - Jaboticabal / São Paulo; Email: ampbantonio@gmail.com

5 Doutor em Solos e Nutrição de Plantas, ESALQ/USP - Universidade de São Paulo, e-mail: jrzanini@fcav.unesp.br
} 
- Fórmula Universal (Darcy-Weisbach) (Equação 1):

$$
h_{f}=f \frac{L}{D} \frac{V^{2}}{2 g}
$$

Sendo essas equações difícil de ser determinadas pelo fato de f não ser facilmente apresentado, uma fórmula aproximada para determinar f é a equação de Swamee-Jain (Equação 2):

$$
f=\frac{0,25}{\left[\log \left(\frac{e}{3,7 D}+\frac{5,74}{R e y^{0,9}}\right)\right]^{2}}
$$

- Fórmula de Hazen-Williams (Equação 3, 4, 5 e 6) (aceito apenas para água em regime turbulento; recomendado para diâmetro maior ou igual a $50 \mathrm{~mm}$ ):

$$
\begin{aligned}
V & =0,355 C D^{0,63} J^{0,54} \\
Q & =0,2788 C D^{2,63} J^{0,54} \\
J & =\frac{6,81 V^{1,85}}{C^{1,85} D^{1,17}}=\frac{10,65 Q^{1,85}}{C^{1,85} D^{4,87}} \\
D & =\frac{Q^{0,38}}{0,615 C^{0,38} J^{0,205}}
\end{aligned}
$$

- Fórmula de Blasius:

$$
h f=7,779 \times 10^{-4} L \frac{Q^{1,75}}{D^{4,75}}
$$

O objetivo do experimento foi calcular a perda de carga principal entre dois pontos de uma mangueira de polietileno em diferentes vazões, utilizando o manômetro de coluna de mercúrio; comparar elas através das equação de Hazen-Williams, Fórmula Universal, equação de Swamme e Jain e equação de Blasius, e traçar gráficos com os dados obtidos.

\section{Relato de Experiência}

Materiais utilizados: - Tubulação de PVC e mangueiras; Registros de controle da vazão; Caixas d'água; Sistema de bombeamento com bomba centrífuga; Manômetros de mercúrio em tubo em U; Balança; Balde; Cronômetro; Tabela de anotação dos resultados na apostila.

Descrição dos procedimentos da aula: 1- Foi conectado, com distância de 10 metros, à mesma altura, em dois pontos uma mangueira com diâmetro menor à mangueira de polietileno da tubulação até cada extremidade do tubo em U de mercúrio, sendo que a perda de carga foi medida entre esses dois pontos da mangueira. 2- Com a caixa d'água cheia, a bomba foi 
ligada, para que bombeasse água para as tubulações e retira da todo ar de dentro das mangueiras. 3- Foi pesado o recipiente de coleta da água vazio, para desconsiderar seu peso do peso final, após coletado certo volume de água. Balde vazio $=1,800 \mathrm{kgf}$. 4 - A vazão foi controlada com uma válvula de gaveta ao final da mangueira. 5- Foram coletados quatro vazões diferentes, anotou-se os limites inferior e superior da coluna do manômetro, o tempo de coleta cronometrado e o volume de água coletado. Depois foram feitos os cálculos de perda de carga ao longo da tubulação.

Os dados obtidos foram anotados na tabela 1, sendo eles a leitura do manômetro (limite inferior e superior), tempo de coleta e volume coletado. Com esses valores foram calculados a vazão e perda de carga: por diferença de pressão; pela equação de HazenWilliams (Equação 5); pela Formula Universal (Equação 1), utilizando a equação de SwameeJain (Equação 2); e pela equação de Blasius (Equação 7).

TABELA 1 - Anotações dos dados coletados em diferentes vazões, 2018.

\begin{tabular}{cccc}
\hline \multicolumn{2}{c}{ Leitura no manômetro $(\mathrm{mm})$} & Tempo de coleta $(\mathrm{s})$ & Volume coletado $\left(\mathrm{m}^{3}\right)$ \\
Inferior & Superior & 64 & 0,0188 \\
10 & 16 & 50 & 0,0225 \\
33 & 25 & 55 & 0,0401 \\
54 & 49 & 49 & 0,0441 \\
\hline
\end{tabular}

Fonte: A autora.

Foram determinados os cálculos da altura da coluna do fluido (2); vazão (3); e a perda de carga determinado $(\mathrm{m} / 100 \mathrm{~m})(4)$ :

$$
\begin{array}{lc}
\multicolumn{1}{c}{\mathrm{h}^{\prime}=\text { inferior }+ \text { superior }} & \mathrm{Hf}=12,596 \mathrm{~h} \text { ' (m.c.fluido) } \\
1+16=17 \mathrm{~mm}=0,017 \mathrm{~m} & \mathrm{hf}=12,596 \times 0,017=\mathrm{hf}=0,2141 \text { (m.c.fluido) } \\
10+25=35 \mathrm{~mm}=0,035 \mathrm{~m} & \mathrm{hf}=12,596 \times 0,035=\mathrm{hf}=0,4408 \text { (m.c.fluido) } \\
33+49=82 \mathrm{~mm}=0,82 \mathrm{~m} & \mathrm{hf}=12,596 \times 0,082=\mathrm{hf}=1,0328 \text { (m.c.fluido) } \\
54+69=123 \mathrm{~mm}=0,123 \mathrm{~m} & \mathrm{hf}=12,596 \times 0,123=\mathrm{hf}=1,5493 \text { (m.c.fluido) }
\end{array}
$$

$\mathrm{Q}=$ volume / tempo

$\mathrm{Q}=0,0188 / 64=2,937 \times \quad \square 10 \rrbracket \wedge(-4)$ $\mathrm{m}^{3} / \mathrm{s}$

Perda de carga determinada $(\mathrm{m} / 100 \mathrm{~m}) \quad \mathrm{J}=(\mathrm{hf} / \mathrm{L}) \times 100(\mathrm{~m} / 100 \mathrm{~m})$ $J=(0,2141 / 10) \times 100=2,14 \mathrm{~m} / 100 \mathrm{~m}$

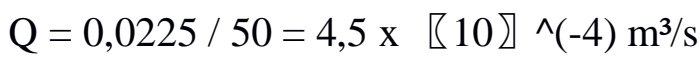
$\mathrm{J}=(0,4408 / 10) \times 100=4,41 \mathrm{~m} / 100 \mathrm{~m}$ $\mathrm{Q}=0,0401 / 55=7,29 \times$ \ $\square 10 \rrbracket \wedge(-4) \mathrm{m}^{3} / \mathrm{s}$ $\mathrm{J}=(1,0328 / 10) \times 100=10,32 \mathrm{~m} / 100 \mathrm{~m}$ $\mathrm{Q}=0,0441 / 49=9,0 \times \quad \square 10 \Omega \wedge(-4) \mathrm{m}^{3} / \mathrm{s}$ 


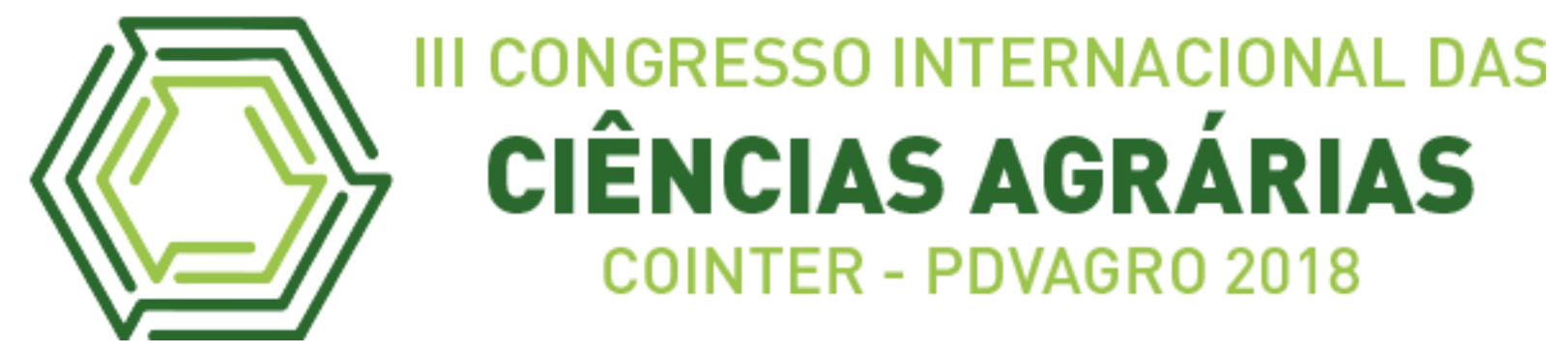

Os valores de perda de carga determinados foram comparados aos dados estimados através das equações de Hazen \& Williams (Equação 5), Fórmula universal - Swamee \& Jain (Equação 1 e 2) e Blasius (Equação 7). Todos os dados obtidos estão alocados na tabela 2.

TABELA 2 - Resultados dos cálculos de perda de carga determinado em manômetro de mercúrio e os métodos de Hazen-Williams, Swamee e Jain, e Blasius, 2018.

\begin{tabular}{|c|c|c|c|c|c|}
\hline \multicolumn{3}{|c|}{ Leitura no manômetro (mm) } & \multirow{2}{*}{$\begin{array}{l}\text { Tempo de coleta } \\
\text { (s) }\end{array}$} & \multirow{2}{*}{ Volume coletado $\left(\mathrm{m}^{3}\right)$} & \multirow{2}{*}{$\mathrm{Q}\left(\mathrm{m}^{3} / \mathrm{s}\right)$} \\
\hline & rior & Superior & & & \\
\hline & & 16 & 64 & 0,0188 & 0,00029 \\
\hline & & 25 & 50 & 0,0225 & 0,00045 \\
\hline & & 49 & 55 & 0,0401 & 0,00073 \\
\hline & & 69 & 49 & 0,0441 & 0,00090 \\
\hline $\mathrm{V}(\mathrm{m} / \mathrm{s})$ & $\begin{array}{c}\text { Rey } \\
\text { (VD/visc) }\end{array}$ & $\begin{array}{l}\text { J Obtido } \\
(\mathrm{m} / 100 \mathrm{~m})\end{array}$ & $\begin{array}{l}\text { Hazen-Williams } \\
(\mathrm{m} / 100 \mathrm{~m})\end{array}$ & $\begin{array}{c}\text { Fórmula Universal - } \\
\text { Swamee e Jain } \\
(\mathrm{m} / 100 \mathrm{~m})\end{array}$ & $\begin{array}{c}\text { Blasius } \\
(\mathrm{m} / 100 \mathrm{~m})\end{array}$ \\
\hline 0,59 & $14.774,62$ & 2,14 & 1,85 & 2,087 & 2,078 \\
\hline 0,91 & $22.691,39$ & 4,41 & 4,093 & 4,464 & 4,403 \\
\hline 1,48 & $36.760,06$ & 10,32 & 9,992 & 10,608 & 10,244 \\
\hline 1,83 & $45.382,79$ & 15,49 & 14,75 & 15,541 & 14,812 \\
\hline
\end{tabular}

Fonte: A autora.

Comparando-se os resultados obtidos, a equação de Hazen \& Williams obteve valores bem inferiores ao valor de perda de carga determinada pela coluna de mercúrio, já para as equações de Swamee \& Jain e Blasius os valores ficaram bem próximo ao determinado.

\section{Considerações}

A equação de Blasius foi a que apresentou menor variação em relação aos valores de perda de carga determinados em coluna de mercúrio, exceto na última vazão, sendo a equação de Swamee \& Jain melhor representada. Essas equações apresentou maior eficiência na determinação dos dados.

\section{Referências}

ANDRADE, A. L. de. Máquinas hidráulicas / AT-087. Paraná, [20--?]. (Apostila). ECO EDUCACIONAL. Roteiro de aula prática: Experimento de perda de carga em acessórios hidráulicos. Florianópolis, 2014.

ZANINI, J. R. Hidráulica - Teoria e Exercícios. Jaboticabal, 2017. (Apostila). 